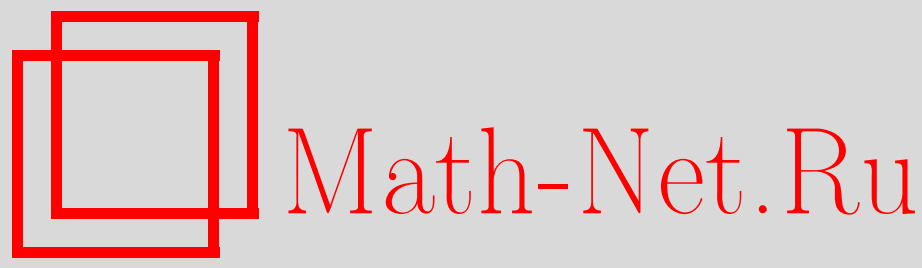

И. В. Маресин, Конформные системы отсчета для лоренцевых многообразий, ТМФ, 2017, том 191, номер 2, 243-253

DOI: https://doi.org/10.4213/tmf9202

Использование Общероссийского математического портала Math-Net.Ru подразумевает, что вы прочитали и согласны с пользовательским соглашением http: //www . mathnet.ru/rus/agreement

Параметры загрузки:

IP : 3.85 .73 .92

26 апреля 2023 г., 11:44:56

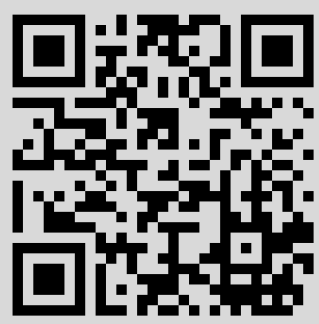


Том 191, № 2

май, 2017

\title{
КОНФОРМНЫЕ СИСТЕМЫ ОТСЧЕТА ДЛЯ ЛОРЕНЦЕВЫХ МНОГООБРАЗИЙ
}

\begin{abstract}
Дано определение конформной системы отсчета - особого класса проекций шестимерного расслоения небесных сфер лоренцева многообразия (или пятимерного пространства твисторов) на трехмерное многообразие. Построен ее пример - конформная компактификация - для пространства Минковского. Дано инвариантное выражение 1-формы на расслоении небесных сфер, порождающей контактную структуру в пространстве твисторов, когда оно гладко. На ориентированном лоренцевом многообразии спинорное соответствие выражено через комплексную структуру на небесных сферах. Доказана теорема о проекции упомянутой 1-формы на послойно нормальное расслоение системы отсчета, следствием которой является уравнение потока времени.
\end{abstract}

Ключевые слова: лоренцевы многообразия, небесные сферы, изотропные геодезические, твисторы, контактная геометрия, линейные расслоения, спиноры, конформная геометрия, световой конус, компактификация Пенроуза.

DOI: https://doi.org/10.4213/tmf9202

\section{1. ВВЕДЕНИЕ}

Твисторный подход к лоренцевым многообразиям основан на описании пятимерного пространства всех светоподобных геодезических $\mathfrak{N}$. В случае пространства Минковского оно может быть вложено в комплексное проективное пространство твисторов, однако в общем (искривленном) случае канонической комплексной структуры на твисторах не существует. Тем не менее каждой точке $x \in X$ соответствует небесная сфера $\mathfrak{S}_{x}$, лежащая в $\mathfrak{N}$, причем $\mathfrak{N}$ наделяется естественной контактной структурой [1]. Небесная сфера $\mathfrak{S}_{x}$ имеет естественную конформную структуру

Работа поддержана грантом РФФИ № 16-01-00117А "Комплексные задачи математической физики" и программой РАН № І.37П "Нелинейная динамика в математических и физических науках", проект № 0014-2015-0037 "Комплексные и алгебро-геометрические методы в задачах нелинейной динамики".

* Математический институт им. В. А. Стеклова Российской академии наук, Москва, Россия. E-mail: qq@superstructure.info 
и изоморфна $\mathbb{C P}^{1}$. Это позволяет описать давно известное твисторное соответствие геометризованным способом, позволяющим обобщение на искривленный случай, свободное от указанного недостатка - невозможности пользоваться комплексной структурой глобально.

Введенное в настоящей статье определение конформной системы отсчета дает описание (четырехмерного) лоренцева многообразия в терминах отображения его небесных сфер в трехмерное многообразие. Известно, что в глобально гиперболическом случае (и в некоторых других) $\mathfrak{N}$ расслаивается над поверхностью Коши $M \subset X$. Тогда вместо сфер - подмногообразий $\mathfrak{N}$ - можно рассмотреть отображения $\mathfrak{S}_{x}$ в $M$ (в неособых случаях - погружения). Вместо поверхности Коши удобно рассмотреть ее обобщение, конформную систему отсчета. Игнорируя технические сложности дифференциальной геометрии на пространстве $\mathfrak{N}$, конформную систему отсчета следует понимать как гладкое отображение из $\mathfrak{N}$, или его части, в произвольное 3 -многообразие $M$, совместимое с контактной структурой, обладающее определенным условием невырожденности (а именно, для всякого $x \in X$ отображение $T \mathfrak{S}_{x} \rightarrow T M$ имеет ранг 2 на непустом открытом подмножестве $\left.\mathfrak{S}_{x}\right)$. В настоящей работе представлено уравнение потока времени, описывающее, как образ неба $\mathfrak{S}_{x}$ зависит от точки $x$ и как это связано с контактной структурой и отношением причинности, но не с топологической точки зрения, а с дифференциальной. При этом показано, что голоморфные сечения линейного расслоения над небесной сферой пригодны для построения спинорного соответствия и "расслоение размера" (с представлением веса $(1 / 2,1 / 2)$ группы $S L(2, \mathbb{C}))$ является естественной областью значения контактной формы на пространстве твисторов.

\section{2. РАССЛОЕНИЕ НЕБЕСНЫХ СФЕР}

2.1. Предварительные сведения. В данном пункте излагаются известные факты о лоренцевых многообразиях, под которыми обычно понимаются псевдоримановы многообразия сигнатуры $(1,3)$, т. е. $(+---)^{1)}$. Настоящая работа требует дополнительной структуры.

ОПРЕДЕЛЕНИЕ 1. Лоренцево многообразие - псевдориманово четырехмерное многообразие с метрикой $g$ сигнатуры $(+---)$ и заданным в каждой точке $x \in X$ направлением времени (т. е. одна из двух компонент связности конуса $\left\{v \in T_{x} X \mid\right.$ $g(v)>0\}$ выбрана как “хронологическое будущее"), причем непрерывным образом.

В работе [1] многообразие $X$, удовлетворяющее этому определению, называется пространством-временем. Все многообразия будут предполагаться гладкими $\left(C^{\infty}\right)$.

ОПРЕДЕЛЕНИЕ 2. Для каждого $x \in X$ граница его конуса "хронологического будущего" в $T_{x} X$ называется световым конусом будущего и обозначается $\mathcal{C}_{x}^{+}$.

ОПРЕДЕЛЕНИЕ 3. Небесная сфера $\mathfrak{S}_{x}$ является базой конуса $\mathcal{C}_{x}^{+}$в $T_{x} X$, а ее точки обозначаются $\mathrm{P} v$, где $v \in \mathcal{C}_{x}^{+} \backslash\{0\}$. Объединение небесных сфер по всем точкам $X$ образует локально тривиальное гладкое расслоение над $X$, обозначаемое через $\mathfrak{S} X$, с функцией проекции х : $\mathfrak{S} X \rightarrow X$.

1) Многие авторы используют метрику $(-+++)$. Разница может влиять на алгебраические аспекты теории, но геометрия остается такой же. 
ЗАмЕчАниЕ 1. Точки небесной сферы понимаются как светоподобные направления (1-подпространства) в $T_{x} X$, а $\mathrm{P}$ - как проективизация $v \mapsto\{\lambda v \mid \lambda \in \mathbb{C}\}$.

ОПРЕДЕЛЕНИЕ 4 . Обозначим через $T_{\mathrm{v}} \mathfrak{S} X$ объединение касательных расслоений $T \mathfrak{S}_{x}$ по всем $x \in X$, иными словами вертикальное подрасслоение $\operatorname{ker} d \mathrm{x}$ в $T(\mathfrak{S} X)$.

ОПРЕДЕЛЕНИЕ 5. Геодезический поток $F X$ - поле 1-подпространств в касательном расслоении $T(\mathfrak{S} X)$ тотального пространства расслоения $\mathfrak{S} X$, задаваемое уравнениями $d x \| v$ (приращение по $x$ коллинеарно $v$ ), $\nabla v=0$, где $v \in \mathcal{C}_{x}^{+} \backslash\{0\}$ - вектор, представляющий точку небесной сферы, а $\nabla$ - связность Леви-Чивиты.

ЗАмечАниЕ 2. Интегрирование потока $F X$ дает "световое" слоение тотального пространства $\mathfrak{S} X$, листами которого являются изотропные (светоподобные) геодезические на $X$, максимально продолженные в обе стороны времени и поднятые на $\mathfrak{S} X$ отображением Р касательных векторов на небесные сферы.

ОПРЕДЕЛЕНИЕ 6. Отношение эквивалентности $\left[\left(x_{1}, \mathrm{P} v_{1}\right)\right]=\left[\left(x_{2}, \mathrm{P} v_{2}\right)\right]$ на точках тотального пространства $\mathfrak{S} X-$ свойство изотропных векторов $v_{1} \in \mathcal{C}_{x_{1}}^{+} \backslash\{0\}$ и $v_{2} \in$ $\mathcal{C}_{x_{2}}^{+} \backslash\{0\}$ лежать на одной изотропной геодезической (т. е. на листе слоения; см. замечание 2).

Из работ [2] и [1] известно, что на $\mathfrak{N}$ - факторпространстве $\mathfrak{S} X$ по введенному выше отношению эквивалентности - имеется естественная контактная структура (когда $\mathfrak{N}$ гладко). В настоящей статье используется версия этого утверждения, приспособленная к факту, что $\mathfrak{N}$ не всегда обладает структурой многообразия.

ОПРЕДЕЛЕНИЕ 7. Выберем гладким способом для каждой точки $w \in \mathfrak{S} X$ по представителю $v \in \mathcal{C}_{x}^{+} \backslash\{0\}$, где $x:=\mathrm{x}(w)$. Тогда $\vartheta=(v . d x)-1$-форма на тотальном пространстве $\mathfrak{S} X$.

Очевидно, данное определение 1-формы имеет смысл лишь с точностью до умножения на положительную функцию. Однако существует глобальное линейное расслоение с помеченной положительной стороной, значения в котором принимает инвариантно определенная форма $\vartheta$. Это построение будет описано в п. 3.2.

ЗАмечАниЕ 3. 1-форма $\vartheta$ гладка и нигде не обращается в нуль. Но $\vartheta$ обращается в нуль как $F X$ (следует из того, что всякое лоренцево изотропное направление ортогонально себе), так и $T_{\mathrm{v}} \mathfrak{S} X$ (следует из его уравнения $\left.d \mathrm{x}=0\right)$. Или иначе: пятимерное расслоение $T(\mathfrak{S} X) / F X$ обладает непрерывным полем однородных гиперплоскостей $\vartheta=0$, содержащих $T \mathfrak{S}_{x}$ для всех $x \in X$, с помеченной положительной стороной.

\section{2. Определение конформной системы отсчета.}

ОПРЕДЕЛЕНИЕ 8. Конформная система отсчета $(\Omega, M, j)$ лоренцева многообразия $X$ задается следующим образом:

1) открытое подмножество $\Omega \subset \mathfrak{S} X$ такое, что всякий его слой $\Omega_{x}:=\Omega \cap \mathfrak{S}_{x}$, $x \in X$ непуст;

2) трехмерное гладкое многообразие $M$;

3) гладкое отображение $j$ из $\Omega$ в $M$ такое, что

$$
\forall w_{1}, w_{2} \in \Omega \quad\left[w_{1}\right]=\left[w_{2}\right] \Rightarrow j\left(w_{1}\right)=j\left(w_{2}\right)
$$


(т. е. отображение постоянно на листах "светового" слоения),

$$
\text { (c) }\left.\forall w \in \Omega \quad \vartheta\right|_{w} \in j^{*}\left(T_{j(w)}^{*} M\right)
$$

(т. е. выделенное поле лучей, представленное формой $\vartheta$ в $T^{*}(\Omega)$, лежит в $j^{*}\left(T^{*} M\right)$, обратном образе кокасательного расслоения), а также

(d) производная $j$ вдоль $T \Omega_{x}$ (слоев $\Omega$ ) не вырождается (т. е. отображение $j_{*}: T_{\mathrm{v}} \Omega \rightarrow T M$ имеет всюду ранг 2$)$.

Образ $\Omega_{x}$ под действием $j$ (погруженная в $M$ поверхность) обозначим $\mathfrak{M}_{x}$ и назовем образом неба.

Пример 1. Пусть $M$ - поверхность Коши в глобально гиперболическом лоренцевом многообразии, $X$ - его часть, лежащая после $M^{2)}, j$ - операция проецирования вдоль изотропных геодезических на твисторное расслоение $S T^{*} M$ (см. [1]) с последующей проекцией на $M$, а $\Omega$ - неособое подмножество $\mathfrak{S} X$, на котором отображение $j_{*}: T_{\mathrm{v}} \mathfrak{S} \rightarrow T M$ имеет ранг 2 . Если всюду на $X$ выполнено условие непустоты $\Omega_{x}$, то $(\Omega, M, j)$ является конформной системой отсчета многообразия $X$.

2.3. Производная образов неба. Определим производную образов неба $\mathfrak{M}_{x}:=$ $j\left(\Omega_{x}\right)$ по $x \in X$. Будем считать дифференциалом отображения $j$ гомоморфизм $d j: T \Omega \rightarrow j^{*}(T M)$ расслоений над $\Omega$, из касательного расслоения к $\Omega$ в обратный образ касательного расслоения к $M$.

ОПРЕДЕЛЕНИЕ 9. В условиях конформной системы отсчета фактор

$$
N_{M} \Omega:=j^{*}(T M) / d j\left(T_{\mathrm{v}} \Omega\right), \quad \text { где } \quad T_{\mathrm{v}} \Omega:=\left.T_{\mathrm{v}} \mathfrak{S} X\right|_{\Omega},
$$

назовем послойно нормальным расслоением.

ЗАмечание 4. Ограничение $N_{M} \Omega$ на $\Omega_{x}$, т. е. "слой" $N_{M} \Omega_{x}=\left.j\right|_{\Omega_{x}} ^{*}(T M) / d j\left(T \Omega_{x}\right)$, послойно нормального расслоения является поднятым на $\Omega_{x}$ нормальным расслоением $N \mathfrak{M}_{x}$ поверхности $\mathfrak{M}_{x}$ в $M$, вне самопересечений, под действием отображения $j: \Omega \rightarrow M$.

ЗАмЕчАНИЕ 5. Понятие нормального расслоения не требует никакой структуры сверх дифференцируемости: касательные векторы объемлющего пространства факторизуются по касательным к погружению.

ОПРЕДЕЛЕНИЕ 10. Для локальной тривиализации проекции х $\Omega \rightarrow X$ (т. е. такой области $U \subset X$, что $U \times \Delta$ представляет подобласть в $\Omega$, где $\Delta$ - стандартный круг в слоях, а х проецирует $U \times \Delta$ на $U)$ и отображения в $M$, заданного как $j: U \times \Delta \rightarrow M$, производная семейства $\mathfrak{M}_{x}$ по $x \in X$ определяется (локально) как гомоморфизм векторных расслоений из $\Delta \times T X$ в соответствующую часть множества $N_{M} \Omega$ :

$$
d \mathfrak{M}_{x}:=\frac{\partial j}{\partial x} / d j(T \Delta),
$$

где “/” означает факторизацию (по образу $T \Delta$ под действием $j$ ). Иными словами, векторы $\frac{\partial j}{\partial x}(\xi) \in j^{*}(T M)$ для всех $(x, z) \in U \times \Delta, \xi \in T_{x} X$ представляют $d \mathfrak{M}_{x}(\xi) \in$ $N_{M} \Omega($ в той же точке $(x, z))$.

${ }^{2)}$ В более общем случае, имеющем отношение к космологии Большого взрыва (пространства Робертсона-Уокера), $M$ может являться конформной границей $X$ (см. [3], разделы 6.8 и 10). 
Локальная тривиализация везде существует и, очевидно, от ее выбора значение производной $d \mathfrak{M}_{x}$ не зависит.

ОПРЕДЕЛЕНиЕ 11. $N \Omega:=T \Omega / T_{\mathrm{v}} \Omega$ - естественная (не зависящая от тривиализации) область определения отображения $d \mathfrak{M}_{x}$ :

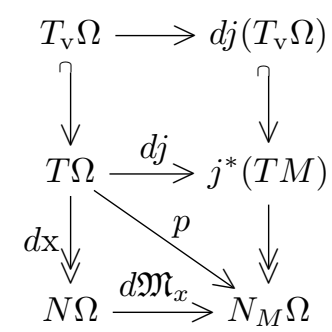

Аналогично для всего $\mathfrak{S} X$ обозначим через $N \mathfrak{S} X:=\mathrm{x}^{*}(T X)=T(\mathfrak{S} X) / T_{\mathrm{v}} \mathfrak{S} X$ поднятое расслоение касательных к $X$ векторов. Кроме того, $d \mathrm{x}$ - соответствующая проекция из $T$ в $N$.

ОПРЕДЕЛЕНИЕ 12 . В тех же условиях определим $p:=d j / d j\left(T_{\mathrm{v}} \Omega\right): T \Omega \rightarrow N_{M} \Omega-$ тавтологическую проекцию $T \Omega$ на послойно нормальное расслоение.

\section{3. ПРИМЕР: ПРОСТРАНСТВО МИНКОВСКОГО}

\section{1. Определение и спинорное соответствие.}

ОПРЕДЕЛЕНИЕ 13. Алгебраическое спинорное соответствие, или преобразование Паули, отображает вектор из $\mathbb{R}^{4}$, заданный компонентами $\left(x^{0}, x^{1}, x^{2}, x^{3}\right)$, в эрмитову $(2 \times 2)$-матрицу ${ }^{3)}$ :

$$
x^{A A^{\prime}}=\frac{1}{2}\left(\begin{array}{cc}
x^{0}+x^{3} & x^{1}+i x^{2} \\
x^{1}-i x^{2} & x^{0}-x^{3}
\end{array}\right) .
$$

ЗАмЕчАниЕ 6. Нетрудно проверить, что всякий изотропный (для метрики $\eta=$ $\left.\left(d x^{0}\right)^{2}-\left(d x^{1}\right)^{2}-\left(d x^{2}\right)^{2}-\left(d x^{3}\right)^{2}\right)$ вектор $x \in \mathbb{R}^{4}$ с условием $x^{0} \geqslant 0$ допускает выражение

$$
x^{A A^{\prime}}=\psi^{A} \bar{\psi}^{A^{\prime}}, \quad \psi \in \mathbb{S}^{1,0}, \quad \text { где } \quad \mathbb{S}^{1,0}:=\mathbb{C}^{2},
$$

и обратно: любой элемент $\psi \in \mathbb{S}^{1,0}$ дает вектор из $\mathcal{C}^{+}$указанным способом.

ОПРЕДЕЛЕНИЕ 14. Пространство Минковского $\mathbb{M}$ - пространство координат $\mathbb{R}^{4}$, снабженное указанной стандартной псевдоевклидовой метрикой.

ЗАмЕчАНИЕ 7 . При этом действию группы $S O^{+}(1,3)$ на $\mathbb{M}$ будет отвечать представление группы $S L(2, \mathbb{C})$ на $\operatorname{Herm}(2)$, заданное через

$$
S L(2, \mathbb{C}) \ni C: x^{A A^{\prime}} \longmapsto C_{L}^{A} x^{L L^{\prime}} \bar{C}_{L^{\prime}}^{A^{\prime}}
$$

(или, в матричном смысле, умножение $x^{A A^{\prime}}$ на $C$ слева и на $C^{*}$ справа), т. е. тензорное произведение двух указанных двумерных представлений на пространствах $\mathbb{S}^{1,0}$ и $\mathbb{S}^{0,1}$ (см. ниже) соответственно.

\footnotetext{
3) Значение положительного множителя здесь несущественно.
} 
ОПРЕДЕЛЕНИЕ 15. Двойственное пространство спиноров $\mathbb{S}_{1,0}^{*}$ есть линейное комплексное пространство, двойственное к $\mathbb{S}^{1,0}$ и снабженное представлением группы $S L(2, \mathbb{C})$ умножением вектор-строк на обратную матрицу $C^{-1}$ справа.

ЗАмЕчАниЕ 8. Все расслоение $\mathfrak{S M}$ имеет естественную тривиализацию, возникающую из тривиализации $T \mathbb{M}$ параллельными переносами. Соответствие между проективизациями конуса $\mathcal{C}^{+}$и пространства $\mathbb{S}^{1,0}$ позволяет также отождествить $\mathfrak{S}$ базу конуса - со сферой Римана $\mathbb{C P}^{1}=\mathbf{P}^{1,0}$. C другой стороны, всякому $\mathrm{P} \psi$, $\psi \in \mathbb{S}^{1,0} \backslash\{0\}$, соответствует однородная прямая $\left\{\varsigma \in \mathbb{S}_{1,0}^{*} \mid \varsigma \psi=0\right\}$, так что проективизации пространств $\mathbb{S}_{1,0}^{*}$ и $\mathbb{S}^{1,0}$ канонически изоморфны.

По причинам, объясняемым ниже, точки небесной сферы пространства Минковского удобно представлять как $\mathrm{P} \varsigma$, где $\varsigma \in \mathbb{S}_{1,0}^{*} \backslash\{0\}$, и отождествлять при этом с комплексными однородными прямыми в $\mathbb{S}_{1,0}^{*}$.

\section{2. Комплексные линейные расслоения.}

ОПРЕДЕЛЕНИЕ 16. Расслоение $\mathcal{O}(k, l), k, l \in \mathbb{Z}$, над $\mathfrak{S}$ есть комплексное линейное расслоение, слоем которого в точке $\mathrm{P} \varsigma, \varsigma \in \mathbb{S}_{1,0}^{*} \backslash\{0\}$, является одномерное пространство однородных комплекснозначных функций бистепени $(k, l)$ на однородной прямой $\{\lambda \varsigma \mid \lambda \in \mathbb{C}\} \subset \mathbb{S}_{1,0}^{*}$. Под однородностью понимается следующее свойство:

$$
\forall f \in \mathcal{O}(k, l)_{\mathrm{P} \varsigma} \quad \forall \lambda \in \mathbb{C}: f(\lambda \varsigma)=\lambda^{k} \bar{\lambda}^{l} f(\varsigma) .
$$

ЗАмЕчАниЕ 9. Всякая однородная функция бистепени $(k, l)$ на всем пространстве $\mathbb{S}_{1,0}^{*}$ задает сечение $\mathcal{O}(k, l)$ путем ограничения на комплексные однородные прямые.

ЗАМЕЧАНИЕ 10 . В частности, $\mathcal{O}(1,0)$ - голоморфное расслоение, причем всякое $\omega \in \mathbb{S}^{1,0}$ задает его голоморфное сечение как линейный функционал на $\mathbb{S}_{1,0}^{*}$. Таким образом, пространство спиноров $\mathbb{S}^{1,0}=\Gamma_{\text {hol }}(\mathcal{O}(1,0))$ - пространство голоморфных сечений.

ОПРЕДЕЛЕНИЕ $17 . \mathbb{S}^{0,1}:=\overline{\mathbb{S}^{1,0}}=\Gamma_{\text {antihol }}(\mathcal{O}(0,1))$ - комплексно-сопряженное пространство спиноров. Кроме того, ему двойственно пространство $\mathbb{S}_{0,1}^{*}$.

ЗАмЕчАНиЕ 11. Как видно из представления, $(2 \times 2)$-матрицы Паули следует понимать как элементы тензорного произведения $\mathbb{S}^{1,0} \otimes \mathbb{S}^{0,1}$, а значит, они задают сечения расслоения $\mathcal{O}(1,1)$.

ОПРЕДЕЛЕНИЕ 18. Под геометрическим спинорным соответствием $\mathrm{sM}_{\mathbb{M}}: \mathbb{M} \rightarrow$ $\Gamma(\mathcal{O}(1,1))$ понимается то же отображение из $\mathbb{M}$ в $\mathbb{S}^{1,0} \otimes \mathbb{S}^{0,1}$, что и в алгебраическом, но его значения интерпретируются как $(1,1)$-однородные неголоморфные многочлены $\varsigma_{A} x^{A A^{\prime}} \bar{\varsigma}_{A^{\prime}}$ на $\mathbb{S}_{1,0}^{*}$ или, что то же самое, сечения $\varsigma_{A} \cdot x^{A A^{\prime}} \cdot \bar{\varsigma}_{A^{\prime}}$ неголоморфного расслоения $\mathcal{O}(1,1)=\mathcal{O}(1,0) \otimes \mathcal{O}(0,1)$ над $\mathfrak{S}$.

Иное название $\mathrm{s}_{\mathbb{M}}-$ иелестиальное преобразование (от англ. celestial transform). В дальнейшем $\mathrm{SM}_{\mathrm{M}}$ будет пониматься также как соответствующее отображение из $\mathbb{M} \times \mathfrak{S}=\mathfrak{S M}$ в тотальное пространство $\mathcal{O}(1,1)$.

ЗАмечаниЕ 12. Два расслоения $\mathcal{O}(1,1)$, построенные над $\mathfrak{S}$ и $\overline{\mathfrak{S}}=\mathbf{P} \mathbb{S}_{0,1}^{*}$, не различаются ничем, кроме комплексной структуры на базах (т. е. канонически изоморфны как комплексные линейные расслоения над поверхностями). Поэтому геометрическое спинорное соответствие выдерживает обмен $\mathfrak{S}$ и $\overline{\mathfrak{S}}$ местами. 
ОПРЕДЕЛЕНИЕ 19. Подрасслоение неотрицательных функций в $\mathcal{O}(1,1)$ обозначим $\mathcal{L}^{+}$. Иначе: $\mathcal{L}^{+}=\{\zeta \cdot \bar{\zeta} \mid \zeta \in \mathcal{O}(1,0)\}$. Содержащее его $\mathbb{R}$-линейное расслоение $\mathbb{R}$-значных $(1,1)$-однородных функций обозначим $\mathcal{L}^{\mathbb{R}}$.

ЗАмЕчАниЕ 13 . Слои $\mathcal{L}^{+}$эквивалентны лучу $[0,+\infty)$ с точностью до умножения на положительные числа. Также легко видеть, что значения определенного выше отображения $\mathrm{S}_{\mathbb{M}}$ являются на самом деле сечениями $\mathcal{L}^{\mathbb{R}}$.

ЗАмЕчАниЕ 14 . Естественным образом определены операции $\zeta \mapsto \zeta \cdot \bar{\zeta}$, обозначаемые $|\cdot|^{2}$ (квадрат модуля) и $|\cdot|:(T \mathfrak{S}=\mathcal{O}(2,0)) \rightarrow \mathcal{L}^{+}-$непрерывные отображения расслоений.

Расслоение $\mathcal{L}^{+}$будет называться расслоением размера, а его сечения - полями размера. Расслоение и сечения $\mathcal{L}^{\mathbb{R}}$ будут называться расслоением и полями размера со знаком.

ЗАмЕчание 15. Представления $S L(2, \mathbb{C})$ на перечисленных пространствах спиноров порождают (согласованное с ними) действие той же группы на небесной сфере, соответствующее накрытию

$$
\{ \pm 1\} \hookrightarrow S L(2, \mathbb{C}) \stackrel{2: 1}{\longrightarrow} P S L(2, \mathbb{C}) \rightarrow 1 .
$$

Оно сохраняет $T \mathfrak{S}$ и $\mathcal{O}(1,0)$ как голоморфные расслоения $(\mathcal{O}(1,1)$ и прочие - как линейные расслоения), а векторные поля и сечения $\mathcal{O}(1,1)$ преобразуются так, как они преобразовались бы просто под действием движений сферы Римана.

ЗАМЕЧАниЕ 16. Согласно определению $\vartheta$ в п. 2.1 для всякого $w \in \mathfrak{S M}, x:=\mathrm{x}(w)$ следует выбрать $v \in \mathcal{C}_{x}^{+} \backslash\{0\}$ - изотропное направление на $\mathbb{M}$, представляющее точку $w \in \mathfrak{S} x$. Запишем $v$ в ковариантной форме (сделаем элементом $\left.T^{*} \mathbb{M}\right)$ через $v_{A A^{\prime}}=$ $\varsigma_{A} \bar{\varsigma}_{A^{\prime}}$. Для каждого касательного вектора $\Xi \in T_{w} \mathfrak{S M}$ имеем $\vartheta(\Xi)=v \xi=\varsigma_{A} \xi^{A A^{\prime}} \bar{\varsigma}_{A^{\prime}}$, $\xi:=\mathrm{x}_{*}(\Xi)$, откуда

$$
\vartheta \quad \propto \quad \varsigma_{A} d \mathrm{x}^{A A^{\prime}} \bar{\varsigma}_{A^{\prime}}: T(\mathfrak{S M}) \rightarrow \mathcal{L}^{\mathbb{R}} .
$$

Символ $\propto$ обозначает здесь равенство с точностью до умножения на положительное число.

\section{3. Построение пуанкаре-инвариантной конформной системы отсчета.}

ПреДЛОЖЕНИЕ 1. Набор $\left(\Omega:=\mathfrak{S M}, M:=\mathcal{L}^{\mathbb{R}}, j:=\mathrm{S}_{\mathbb{M}}\right)$ составляет конформную систему отсчета для $\mathbb{M}$.

ЗАмечАниЕ 17 . Тотальное пространство расслоения $\mathcal{L}^{\mathbb{R}}$ представимо как $\mathfrak{S} \times \mathbb{R}$, а все $\mathfrak{M}_{x}$ - графики сечений $\varsigma_{A} \cdot x^{A A^{\prime}} \cdot \bar{\varsigma}_{A^{\prime}}$.

ДокАЗАТЕЛЬСТво ПРЕДЛОЖЕНИЯ 1. Очевидно, отображение, переводящее небесные сферы в графики сечений, удовлетворяет условию (d) из определения конформной системы отсчета. Условие (f) выполнено в силу того, что $\mathrm{s}_{\mathbb{M}}$-образ сферы $\mathfrak{S}_{v}$, где $v$ - изотропный вектор, касается нулевого сечения в точке сферы, соответствующей направлению Р $v$. Для доказательства условия (c) проще всего исследовать $w \in \mathfrak{S}_{0} \mathbb{M}$, что не ограничивает общности, поскольку параллельные переносы $\mathbb{M}$ соответствуют послойному сложению тотального пространства $\mathcal{L}^{\mathbb{R}}$ (и образов неба в нем) с фиксированным гладким сечением. В силу упомянутой в п. 3.1 тривиальности $\mathfrak{S M}$ имеем $T_{w} \mathfrak{S M}=T_{w} \mathfrak{S}_{0} \oplus T_{0} \mathbb{M}$, где $T_{w} \mathfrak{S}_{0} \subset \operatorname{ker} \vartheta$, причем $j_{*}\left(T_{w} \mathfrak{S}_{0}\right)$ состоит 
из векторов, касательных к $\mathfrak{M}_{0}$ (нулевому сечению $\mathcal{L}^{\mathbb{R}}$ ). Выберем аналогично (1) такое $\varsigma \in \mathbb{S}_{1,0}^{*} \backslash\{0\}$, что $\mathrm{P} \varsigma=w$. Прямое вычисление

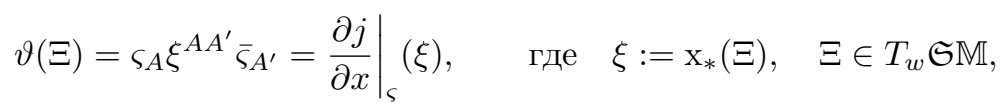

показывает, что искомый образ формы $\left.\vartheta\right|_{w}$ под действием $j$ есть просто проекция пространства $T_{\left(\mathrm{P}_{\varsigma}, 0\right)} \mathcal{L}^{\mathbb{R}}$ на вертикальное (касательное к слоям $\mathcal{L}^{\mathbb{R}}$ ) направление. Образ формы $\vartheta$ в произвольной точке $w \in \mathfrak{S M}, x:=\mathrm{x}(w)$ будет отличаться проецированием вдоль касательной к графику $\varsigma_{A} \cdot x^{A A^{\prime}} \cdot \bar{\varsigma}_{A^{\prime}}=\mathrm{S}_{\mathbb{M}} x$ вместо нулевого сечения.

Прямая интерпретация построенной системы отсчета: тотальное пространство $\mathcal{L}^{\mathbb{R}}$ есть пространство изотропных гиперплоскостей в $\mathbb{M}$, а $j$-прообраз элемента из $\mathcal{L}^{\mathbb{R}}$ задается постоянным световым направлением в $\mathfrak{S}$ и гиперплоскостью в $\mathbb{M}$. Выбрав произвольно $\mathrm{P} \varsigma \in \mathfrak{S}$ и $\left.\chi \in \mathcal{L}^{\mathbb{R}}\right|_{\text {Р }}$, получим множество х $\left(j^{-1}(\chi)\right)$ всех точек $\mathbb{M}$, $\mathrm{SM}_{\mathbb{M}}$-образы небесных сфер которых проходят через $\chi$, как гиперплоскость - решение линейного уравнения $\varsigma_{A} x^{A A^{\prime}} \bar{\varsigma}_{A^{\prime}}=\chi(\varsigma)$ на $x^{A A^{\prime}}$. Из наличия естественного действия группы Пуанкаре в $\mathcal{L}^{\mathbb{R}}$ следует, что таким способом задаются все изотропные гиперплоскости в $\mathbb{M}$.

Указанные изотропные гиперплоскости можно также понимать как световые конусы бесконечно удаленных точек, а само трехмерное тотальное пространство $\mathcal{L}^{\mathbb{R}}-$ как часть $\mathcal{I}$ конформной компактификации $\widehat{\mathbb{M}}$ пространства Минковского ${ }^{4)}$, что сближает настоящий пример с данным в п. 2.2 и будет обосновано в п. 3.4.

3.4. Интерпретация через твисторное соответствие. Многообразие световых прямых пространства $\widehat{\mathbb{M}}$ допускает известное описание как пространство проективных твисторов (изотропных) $\mathbf{P N} \subset \mathbf{P}\left(\mathbb{S}_{0,1}^{*} \times \mathbb{S}^{1,0}\right) \simeq \mathbb{C P}^{3}$. $\mathbf{N}$ задается через $\bar{\pi}_{L} \omega^{L}+\pi_{L^{\prime}} \bar{\omega}^{L^{\prime}}=0$ [6] и является гладкой вещественной гиперповерхностью в $\mathbb{S}_{0,1}^{*} \times \mathbb{S}^{1,0}$, а $\mathbf{P N}$ - в соответствующем комплексном проективном пространстве. Если ограничиться собственно пространством Минковского $(X:=\mathbb{M})$, то $\mathfrak{N}=\mathbf{P N}_{\text {a }} \subset$ $\mathbf{P N}$ (далее называемое афбинной частью), причем $\left[\mathfrak{S}_{x}\right]$ задано следующим уравнением [6]:

$$
\omega^{L}=i x^{L L^{\prime}} \pi_{L^{\prime}}
$$

где $\mathbb{S}_{0,1}^{*} \ni \pi=\bar{\varsigma}-$ комплексные проективные координаты в слоях $\overline{\mathfrak{S M}} \mathbf{M} . \mathbf{N}_{\text {a }}$ же есть объединение всех решений уравнения (2) для всех $x \in \mathbb{M}$, и $\mathbf{N}_{\mathrm{a}}=\mathbf{N} \backslash\{\pi=0\}$. Гладкость отображения [·]: $\mathfrak{S M} \rightarrow \mathbf{P N}_{\text {a }}$ очевидна.

ОПРЕДЕЛЕНИЕ 20. Твисторной сверткой $\tau: \mathbf{P}\left(\mathbb{S}_{0,1}^{*} \backslash\{0\} \times \mathbb{S}^{1,0}\right) \rightarrow \mathcal{O}(1,1)$ называется отображение, переводящее точку $\mathrm{P}(\pi, \omega)$ в элемент тотального пространства расслоения $\mathcal{O}(1,1)$ над $\overline{\mathfrak{S}}$ (совпадающего с $\mathcal{O}(1,1)$ над $\mathfrak{S})$, заданный точкой Р $\pi$ на базе и "комплексным размером" $\lambda \pi \mapsto-i|\lambda|^{2} \bar{\pi}_{L} \omega^{L}, \lambda \in \mathbb{C}$.

ЗАмЕЧАНИЕ 18. Для корректности определения твисторной свертки требуется, чтобы указанная $(1,1)$-однородная функция на $\{\lambda \pi\}$, зависящая от пары $(\pi, \omega)$ как от параметра, не менялась при умножении проективных координат на ненулевое комплексное число, что, очевидно, выполняется.

4) C конформной компактификацией $\mathbb{M}$ можно ознакомиться в [4] (гл.9) и [5]. Более общее построение конформной границы лоренцева многообразия приведено в [3] (раздел 6.8). 


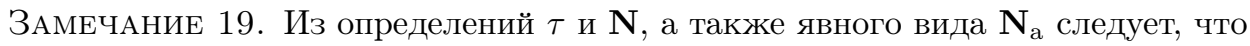

$$
\mathbf{N}_{\mathrm{a}}=\left\{\left(\pi \in \mathbb{S}_{0,1}^{*} \backslash\{0\}, \omega \in \mathbb{S}^{1,0}\right) \mid \tau(\pi, \omega) \in \mathcal{L}^{\mathbb{R}}\right\}
$$

(иначе говоря, $\left.\mathbf{P} \mathbf{N}_{\mathrm{a}}=\tau^{-1}\left(\mathcal{L}^{\mathbb{R}}\right)\right)$. Очевидно, $\tau$-прообраз всякого элемента $\mathcal{L}^{\mathbb{R}}$ есть аффинная часть проективной прямой в $\mathbf{P} \mathbf{N}$ (или плоскости в $\mathbf{N}$ ), но с постоянным Р $\pi$, в отличие от задаваемых уравнением (2).

ЗАмЕчАниЕ 20. Введенное в п. 3.3 отображение $j$ совпадает с композицией проекции $[\cdot]: \mathfrak{S M} \rightarrow \mathbf{P N}_{\mathrm{a}}$ с твисторной сверткой $\tau$ и выводится сворачиванием выражения (2) с $-i \bar{\pi}_{L}$. Таким образом, каждый $\tau$-прообраз элемента $\chi \in \mathcal{L}^{\mathbb{R}}$ задает бесконечно удаленную точку $\widehat{\mathbb{M}}$, в которой пересекаются световые прямые, составляющие изотропную гиперплоскость $\mathrm{x}\left(j^{-1}(\chi)\right)$ в $\mathbb{M}$.

ЗАмечАниЕ 21. Из выражения $j$ через $\tau$ и того факта, что проективная прямая $\omega=0$ есть $\left[\mathfrak{S}_{0}\right] \subset \mathbf{P N}_{\mathrm{a}}$, получаем, аналогично предложению 1 , что контактная форма на $\mathbf{P N}_{\mathrm{a}}$, ограниченная на $\left[\mathfrak{S}_{0}\right]$, допускает выражение

$$
\left.\vartheta\right|_{\left[\mathfrak{S}_{0}\right]}=\left.d \tau\right|_{\{\omega=0\}}=-\left.i \bar{\pi}_{L} d \omega^{L}\right|_{\{\omega=0\}} .
$$

Ввиду однородности $\tau$ выражение $d \tau$ корректно определяет отображение $\left.T \mathbf{P} \mathbf{N}_{\mathrm{a}}\right|_{\left[\mathfrak{S}_{0}\right]} \rightarrow$ $\mathcal{O}(1,1)$.

Нетрудно также показать, что на всем $\mathbf{N}_{\mathrm{a}}$

$$
\vartheta_{\mathbf{N}_{\mathrm{a}}}=-i\left(\bar{\pi}_{L} d \omega^{L}+\bar{\omega}^{L^{\prime}} d \pi_{L^{\prime}}\right) .
$$

\section{4. УРАВНЕНИЕ ПОТОКА ВРЕМЕНИ}

В общем случае лоренцева многообразия необходимо рассматривать расслоения над разными небесными сферами. Через $\mathcal{O}_{x}(1,0), \mathcal{L}_{x}^{+}$и т. п. обозначим соответственные расслоения над небесной сферой $\mathfrak{S}_{x}$. Аналогично (с нижним индексом $x$ ) обозначены зависящие от $x$ пространства спиноров.

ЗАмЕчАниЕ 22. Нетрудно видеть, что обратный образ $\mathrm{x}^{*}\left(T_{x} X\right)=N \mathfrak{S}_{x}$ касательного пространства есть $\mathfrak{S}_{x} \times T_{x} X$, что после замены базы и слоя местами превращается в $\mathfrak{S}\left(T_{x} X\right)$. Причем пространство $\mathfrak{S}\left(T_{x} X\right)$ изоморфно изученному в разделе 3 пространству $\mathfrak{S M}$ и изоморфизм определен с точностью до действия группы Лоренца.

ОПРЕДЕЛЕНИЕ 21. Обозначим через $\mathcal{L}^{\mathbb{R}} X$ объединение расслоений размера со знаком $\mathcal{L}_{x}^{\mathbb{R}}$ по всем $x \in X$ - гладкое линейное расслоение над $\mathfrak{S} X$. Аналогично $\mathcal{L}^{+} X \subset \mathcal{L}^{\mathbb{R}} X$ - объединение расслоений размера $\mathcal{L}_{x}^{+}$по всем $x \in X$.

ОПредЕЛЕНиЕ 22. Отображение s: $N \mathfrak{S} X \rightarrow \mathcal{L}^{\mathbb{R}} X$ определяется послойно через $\mathrm{s}_{T_{x} X}: N \mathfrak{S}_{x} \rightarrow \mathcal{L}_{x}^{\mathbb{R}}$ для всех $x \in X$, где $\mathrm{s}_{T_{x} X}$ идентично $\mathrm{s}_{\mathbb{M}}$, введенному в п. 3.2 .

ЗАмечАниЕ 23. При выбранной (произвольно) ориентации на $T_{x} X$ корректность определения отображения $\mathrm{s}_{T_{x}}$ с следует из $S L(2, \mathbb{C})$-инвариантности пространства $\mathbb{S}_{x}^{1,0}$ и вида соответствующего представления группы в $\mathcal{O}_{x}(1,1)$. Перемена ориентации на $T_{x} X$ переставляет $\mathfrak{S}_{x}$ и $\varsigma_{A} \in \mathbb{S}_{x}^{1,0}$ с $\overline{\mathfrak{S}}_{x}$ и $\pi_{A^{\prime}} \in \mathbb{S}_{x}^{0,1}$ соответственно, но $\mathcal{L}_{x}^{\mathbb{R}}$ и отображение расслоения $N \mathfrak{S}_{x}$ в $\mathcal{L}_{x}^{\mathbb{R}}$ от этого не меняются. 
Теперь мы способны заменить определение формы $\vartheta$, данное в п. 2.1, на следующее

ОПРЕДЕЛЕНИЕ 23. $\vartheta:=\mathrm{s} \circ d \mathrm{x}-1$-форма на тотальном пространстве $\mathfrak{S} X$ со значениями в $\mathcal{L}^{\mathbb{R}} X$, задаваемая композицией горизонтальной проекции $d \mathrm{x}: T(\mathfrak{S} X) \rightarrow$ $N \mathfrak{S} X$ с введенным выше отображением s.

ЗАмечание 24. "Новая" форма $\vartheta: T \Omega \rightarrow \mathcal{L}^{\mathbb{R}} X$ и $\mathbb{R}$-значная форма, определенная в п. 2.1 с точностью до положительного множителя, совпадают как ориентированные конаправления на $\mathfrak{S} X$.

ЗАмечАниЕ 25. Непосредственно из определения $\vartheta$ следует, что $\mathrm{s}=d \mathrm{x}_{*}(\vartheta)$.

Теорема 1. Для любой конформной системы отсчета $(\Omega, M, j)$ лоренцева многообразия $X$ проекиия $p$ (см. n. 2.3) удовлетворяет

$$
\operatorname{ker} p=\left.\operatorname{ker} \vartheta\right|_{\Omega} \text {. }
$$

ДокАЗАТЕЛЬство. В соответствии с определением конформной системы отсчета ядро отображения $d j$ в $T \Omega$ трехмерно, целиком лежит в $\operatorname{ker} \vartheta$ и имеет нулевое пересечение с $T_{\mathrm{v}} \Omega$, т. е. $\left.\operatorname{ker} \vartheta\right|_{\Omega}=\operatorname{ker} d j \oplus T_{\mathrm{v}} \Omega$, а над точками $x \in X$ имеет место $T_{\mathrm{v}} \Omega_{x}=T \Omega_{x}$. Поскольку поднятое нормальное расслоение $N_{M} \Omega_{x}$ - область значений $p$ над $\Omega_{x}-$ получается из $\left.j\right|_{\Omega_{x}} ^{*}(T M)$ факторизацией по образам $d j\left(T \Omega_{x}\right)$ небесных направлений, теорема доказана.

СлЕДСТВИЕ 1. Отображсение $\mathrm{MM}_{x}$ (производная образов неба по $x$ ) обращает в нуль dx-проекиию $\operatorname{ker} \vartheta$, и только ее (см. диаграмму в определении 11).

СлЕДСТвиЕ 2. Прямой образ 1-формы $\vartheta$ под действием отображения $p$ корректно определен и нигде на $\Omega$ не обращается в нуль, задавая изоморфизм расслоений $\mathcal{L}^{\mathbb{R}} \Omega:=\left.\mathcal{L}^{\mathbb{R}} X\right|_{\Omega}$ и $N_{M} \Omega$.

ТеОрема 2. Пусть $X$ - ориентированное многообразие с конформной системой отсчета, удовлетворяющее условиям теоремь 1. Определим, опираясь на следствие 2, гомоморфизм линейньх расслоений $a_{\vartheta}:=p_{*}(\vartheta)^{-1}: \mathcal{L}^{\mathbb{R}} \Omega \rightarrow N_{M} \Omega^{5)}$. Тогда производная (см. п. 2.3) семейства $\left\{\mathfrak{M}_{x}\right\}=\left\{j\left(\Omega_{x}\right)\right\}$ будет задаваться так называемым уравнением потока времени:

$$
d_{A A^{\prime}} \mathfrak{M}_{x}=a_{\vartheta}\left(\varsigma_{A} \cdot \pi_{A^{\prime}}\right) \quad \Leftrightarrow \quad p_{*}(\vartheta) \cdot d_{A A^{\prime}} \mathfrak{M}_{x}=\varsigma_{A} \cdot \pi_{A^{\prime}}
$$

где $\varsigma_{A}$ и $\pi_{A^{\prime}}$ - комплексно-сопряженные друг другу базисы в $\mathbb{S}_{x}^{1,0}$ u $\mathbb{S}_{x}^{0,1}$ (или, что то же самое, координаты в $\left.\mathbb{S}_{x 1,0} u \mathbb{S}_{x 0,1}\right)$ соответственно, в которых записано алгебраическое спинорное соответствие для дифберенциала $d_{A A^{\prime}} \mathfrak{M}_{x}$.

ДокАЗАТЕЛЬСтво. Из тождества $p=d \mathfrak{M}_{x} \circ d \mathrm{x}$, приведенного на диаграмме в определении 11 , и определения $\vartheta$ выводим $p_{*}(\vartheta)=\left(d \mathfrak{M}_{x}\right)_{*}(\mathrm{~s})$. А равенство $\mathrm{s} \xi=$ $\varsigma_{A} \cdot \xi^{A A^{\prime}} \cdot \pi_{A^{\prime}}$ справедливо по построению, где $\xi \in T_{x} X$, откуда и следует утверждение теоремы.

\footnotetext{
${ }^{5)}$ Иначе говоря, $a_{\vartheta}=\vartheta_{*}(p)$ и решение уравнения $a_{\vartheta} \circ \vartheta=p$.
} 


\section{5. ДРУГИЕ РАЗМЕРНОСТИ ПРОСТРАНСТВА}

В качестве обобщения лоренцевых многообразий можно рассмотреть псевдоримановы многообразия сигнатуры $(1, d)$, т. е. один плюс и $d$ минусов (где $d$ - натуральное число), с заданным в каждой точке направлением времени. Содержание раздела 2 применимо к данному случаю с очевидными изменениями, касающимися размерности: небесная “сфера" становится $S^{d-1}, \Delta-(d-1)$-мерный шар, и т. д. Комплексная алгебра из раздела 3 не допускает очевидного обобщения, поскольку лишь для случая $(1,3)$ спинорная группа изоморфна комплексной специальной линейной группе. Тем не менее пространство $\mathbb{M}^{1+d}$ допускает и конформную компактификацию, и построенную в п. 3.3 инвариантную систему отсчета, дающую возможность определить расслоение размера $\mathcal{L}^{\mathbb{R}} X$ как область значений контактной формы. Теорема 1 также остается верной для любого $d$.

Благодарности. Автор признателен А. Г. Сергееву за помощь при подготовке работы к публикации.

\section{Список литературы}

[1] V. Chernov, S. Nemirovski, "Legendrian links, causality, and the Low conjecture", Geom. Funct. Anal., 19:5 (2010), 1320-1333, arXiv: 0810.5091.

[2] C. R. LeBrun, "Twistors, ambitwistors and conformal gravity", Twistors in Mathematics and Physics, London Mathematical Society Lecture Note Series, 156, eds. T. N. Bailey, R. J. Baston, Cambridge Univ. Press, Cambridge, 1990, 71-86.

[3] S. W. Hawking, G. F. R. Ellis, The Large Scale Structure of Space-Time, Cambridge Univ. Press, Cambridge, 1973.

[4] Р. Пенроуз, В. Риндлер, Спиноры и пространство-время. Спинорные и твисторные методы в геометрии пространства-времени, т. 2, Мир, М., 1988.

[5] В. С. Владимиров, А.Г. Сергеев, “О компактификации пространства Минковского и комплексном анализе в трубе будущего", Ann. Polon. Math., 46:1 (1985), 439-454.

[6] Р. Пенроуз, "Твисторная программа”, Твисторы и калибровочные поля, Мир, М., 1983, $13-24$. 